\title{
Characteristics of Oral Tongue and Base of the Tongue Cancer: A Hospital Cancer Registry Based Analysis
}

\author{
Manigreeva Krishnatreya ${ }^{1 *}$, Pintu Nandy ${ }^{1}$, Tashnin Rahman ${ }^{2}$, Jagannath Dev \\ Sharma $^{1}$, Anupam Das ${ }^{2}$, Amal Chandra Kataki ${ }^{1}$, Ashok Kumar Das ${ }^{2}$, Rajjjyoti \\ $\operatorname{Das}^{2}$
}

\begin{abstract}
Background: Tongue cancer is one of the leading sites of cancer in our population. Aim: To evaluate the socio-demographic profiles and stages at diagnosis of oral tongue (OT) and base of tongue (BT) cancers, and identify any possible variations in characteristics. Materials and Methods: A retrospective analysis was conducted on tongue cancer cases, divided into OT and BT, registered at the hospital cancer registry of North-East India during January 2010 to May 2013. Cases were analyzed for age, gender, residential status and different levels of education for patients, the stage at diagnosis and presence of distant metastasis. Results: A total of 1,113 cases of tongue cancers were registered, 846(76.1\%) of BT and 267(23.9\%) of OT. While 33.9\% of BT cancer patients were above 65 years of age, the figure for OT cancers was $18.4 \%$, stages III and IV accounting for $90.8 \%$ and $77 \%$, respectively. The relative risk for distant metastasis in OT cancers was $3.3(95 \%$ CI $1.08-10.1, p=0.03)$. Conclusions: In the subsites of tongue cancers in our population, the majority arose from the base of tongue, these tending to occur in older individuals and presenting at late stage.
\end{abstract}

Keywords: Base of tongue - cancer - disparities - oral tongue - age dependence

Asian Pac J Cancer Prev, 16 (4), 1371-1374

\section{Introduction}

Tongue cancer is one of the common cancers in users of chewable tobacco and alcohol. Of different ways of tobacco consumption like smoking and smokeless tobacco use, the habit of chewable tobacco usage is prevalent in the population of this part of the country (Krishnatreya et al., 2014). In addition to risk factors like tobacco and alcohol there is now a growing interest in the role of human papilloma virus (HPV) infection in cancers of the oral cavity and tongue in particular. Additionally disparities exist amongst our population from lower socio-economic strata who are most vulnerable because of higher exposure to tobacco (Coelho, 2012).

More recently, it has been shown that there are disparities amongst races/ethnic groups in the occurrence of HPV associated tongue and oropharyngeal cancers (Chaturvedi, 2014). The tongue can be divided into 2 parts based on the topography, namely, the oral tongue (OT) located in the oral cavity and the base of tongue (BT) which is anatomically and for clinical purposes considered to be in the oropharynx. The common histology seen in tongue cancers is squamous carcinoma, but other variants of epithelial malignancies are also seen. Worldwide the age adjusted incidence rates (AAR) for tongue cancer is not so significant in relation to other cancers (Ferlay et al., 2014). The AAR of tongue cancer in our population for both males and females is amongst the highest in the world (National Cancer Registry Programme, 2013). The aims of the present study were to evaluate the socio-demographic and stages at diagnosis of OT and BT cancers, and identify any possible variations in its characteristics.

\section{Materials and Methods}

This retrospective study was done on the data of tongue cancer patients registered at a hospital based cancer registry in North-East India. The tongue cancer cases diagnosed during the period of January 2010 to May 2013 were included in the analysis. Cases of tongue cancers were identified by International Statistical Classification for Diseases, 10th revision (ICD-10) coding on the data set $(\mathrm{C} 01=$ base of tongue and $\mathrm{C} 02=$ oral tongue $)$. Sociodemographic analysis was conducted for age, gender, residential statuses and different levels of education for patients with tongue cancers (OT and BT groups separately). The clinical characteristic for the present study was based on the stage at diagnosis and presence of distant metastasis. Residential status was either urban or rural residents. 
Age group

The age-groups for the present analysis were centered on the truncated age group used by the National Cancer Registry Programme of India for truncated incidence rates which is 35-64 years of age. So, the different age groups for the present study were $<35$ years, 35-44, 45-54,55-64 and 65 years and above.

\section{Educational levels}

Broadly the classifications of education according to the National Cancer Registry Programme of India are; not applicable (for children below 5 years), illiterates (patients who are unable to read or write), literates (patients who are able to read and write their names), school or high school levels (primary, middle, secondary and technical education), technical education, and college and above. Educational levels of all patients were clustered into 3 groups for the present study; illiterates (unable to read or write), qualified (school or high school level education), and highly qualified (college and above).

\section{Stage at diagnosis}

The staging of tongue cancer was done according to the American Joint Committee on Cancer Classification (AJCC) (Edge et al., 2010).

\section{Statistical analysis}

Results are presented as percentages and descriptive statistics was calculated up to single decimal place. The test of independence was done by Chi square test. $\mathrm{p}<0.05$ was considered as statistically significant. The analysis was done using Epi-Info 3.5.1.

\section{Results}

In the present retrospective analysis, there were 1113 cases of tongue cancers. Of which, 846(76.1\%) were cancers of the BT and 267(23.9\%) were that of OT cancers. The distribution of cases for males and females are shown in Table 1. The median age for cancers of the BT and OT were 58 years and 50 years respectively. Majority of cases of BT cancer occurred above 65 years $(287 / 846,33.9 \%)$ followed by, in the age group of 55-64 years $(236 / 847,27.8 \%)$, and for OT cancers majority were seen in the age group of 45-54 years $(71 / 267,26.5 \%)$ followed by in the age group of 55-64 years (70/267,26.2\%) (Figure1). Figure 2 shows the trend in the occurrence of OT and BT cancers in both the genders across various age-groups. $80.9 \%$ and $74.2 \%$ of cases reported for cancers of BT and OT respectively were from rural areas (Table 1). Majority of patients with BT and OT cancers were qualified (56.9\%), followed by illiterates in $39.2 \%$ and $28.8 \%$ of BT cancer and OT cancer patients respectively.

The information on stage was present in 783 records and 236 records of BT and OT cancer respectively. The stage at diagnosis for BT cancer was, stage I in 1\% (8/783), stage II in $8.2 \%(64 / 783)$, stage III in $43.4 \%$ (340/783) and stage IV in $47.4 \%(371 / 783)$ of patients (Table 2$)$. The different stages for OT cancer were stage I in 3.8\% (9/236), stage II in $19.5 \%$ (46/236), stage III in $28.4 \%$ (67/236) and
Table 1. The Table Shows the Socio-demographic characteristics of Tongue Cancers

\begin{tabular}{lccc}
\hline Variables & $\begin{array}{c}\text { Base of Tongue } \\
\#(\%)\end{array}$ & $\begin{array}{c}\text { Oral Tongue } \\
\#(\%)\end{array}$ & p value \\
\hline Gender & $671(79.3)$ & $192(71.9)$ & 0.011 \\
Male & $175(20.7)$ & $75(28.1)$ & \\
Female & 58 years & 50 years & - \\
Median Age & {$[$ Range: $24-96]$} & Range: 5-90] & \\
& & & \\
Residential Status & & $198(74.2)$ & 0.019 \\
Rural & $684(80.9)$ & $69(25.8)$ & \\
Urban & $162(19.1)$ & & 0.000 \\
Education Level & & $77(28.8)$ & \\
Illiterate & $332(39.2)$ & $152(56.9)$ & \\
Qualified & $481(56.9)$ & $38(14.2)$ & \\
Highly Qualified & $33(3.9)$ & &
\end{tabular}

\# = Numbers, $\%=$ Percentage

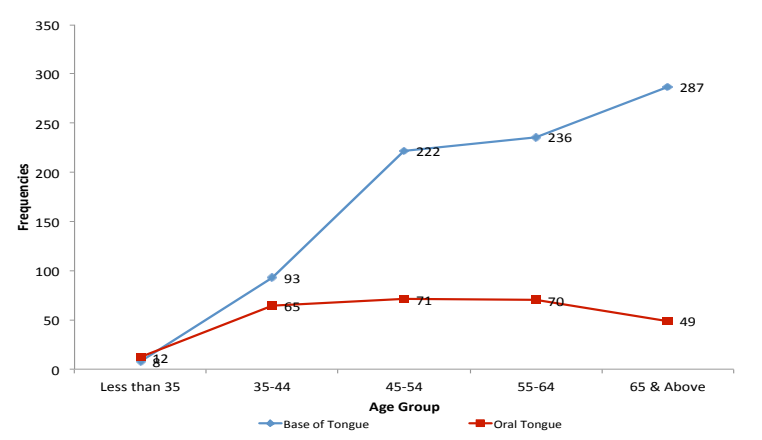

Figure 1. It Shows the Age Group Distribution in Base of Tongue and Oral Tongue Cancer

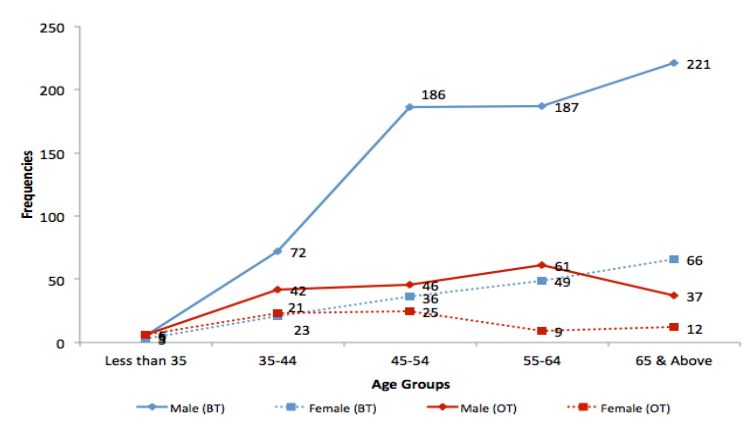

Figure 2. It Shows the Distribution of Age Group and Gender for Base of Tongue and Oral Tongue Cancer

Table 2. Data for the Stage at Diagnosis for Base of Tongue and Oral Tongue Cancers

\begin{tabular}{lcc}
\hline & Base of tongue \# $(\%)$ & Oral tongue \# $(\%)$ \\
\hline Stage I & $8(1.0)$ & $9(3.8)$ \\
Stage II & $64(8.2)$ & $46(19.5)$ \\
Stage III & $340(43.4)$ & $67(28.4)$ \\
Stage IV & $371(47.4)$ & $114(48.3)$ \\
\hline
\end{tabular}

\# = Numbers, $\%=$ Percentage

Table 3. Data for the Association of Metastasis with Oral Tongue Cancers

\begin{tabular}{lcc}
\hline & Oral tongue & Base of tongue \\
\hline M+ & 6 & 6 \\
M- & 230 & 777 \\
\hline
\end{tabular}

*M+ = Metastasis present, M- = Metastasis absent 
Characteristics of Oral Tongue and Base of the Tongue Cancer - A Hospital Cancer Registry Based Analysis

stage IV was seen in $48.3 \%$ (114/236) patients (Table 2). Distant metastasis was seen in $2.5 \%(6 / 236)$ OT cancer and $0.8 \%$ of cases of BT (6/783) cancer. On estimating the relative risk (RR) of distant metastasis for OT cancer it showed RR of 3.3 (95\% confidence interval at 1.08 to 10.1, z statistic was $2.0, \mathrm{p}=0.03$ ) as shown on Table 3 .

\section{Discussion}

The AAR of tongue cancer amongst the leading sites in the population of this region ranges from 2.5 to 9.4 per one lakh population in males and 2.9 to 3.2 per one lakh population of females (National Cancer Registry Programme, 2013). This AAR is calculated combining the BT and OT cancers as one entity. In our analysis, $76 \%$ of cancers of tongue were BT cancer. OT cancer which is likely to be seen due to the use of chewable tobacco and poor oral hygiene was seen in $24 \%$ of cases of tongue cancer in this analysis. There are evidences to associate HPV infection with oral tongue cancers (Elango et al., 2011; Ramshankar et al., 2014). The association of oropharyngeal cancers with HPV infection and more so in the tonsils has also been established (Herrero et al., 2003; Kreimer et al., 2005). More recently though, it has been shown that partners of patients with HPV positive oropharyngeal cancer do not seem to have elevated oral HPV infection compared with the general population ( D'Souza et al., 2014). This assumes significance in view of the fact that in the present analysis; males were 3.8 (M:F ratio) times more affected with $\mathrm{BT}$ cancer and 2.5 times more affected in OT cancers and so, in the likely event of association of tongue caner with HPV infection, it would not result in additional risk of transmission to the female partner to develop tongue cancer.

The habit of betel quid use and tobacco chewing is rampant in rural areas of this region. In an earlier study by Balaram et al., (1995) they reported high incidence of HPV infection in tobacco chewers and betel quid users. As shown in our study almost $81 \%$ of BT cancer patients and $75 \%$ of OT cancer patients were from rural areas. So, the likelihood of co-existence of HPV infection in patients due to tobacco and betel quid users is high in our population. Though in the present analysis both BT and OT cancers were predominantly seen in rural residents but, there was a bias towards the BT cancers for rural residences $(\mathrm{p}<0.05)$. Krishnamurthy and Ramshankar (2013) have shown that majority of early staged oral tongue cancer was seen in the age group of 51-70 years. Our analysis of all stages of OT cancer has also showed that $52.7 \%$ of cases were seen in the age of 45-64 years. However, for BT cancers, most of the cases were seen after 55 years $(61.6 \%)$ and significant number of cases was observed after the cut off age for truncated incidence rates used by NCRP. Also, over 45 years of age there was a sharp increase in the number of cases in males for BT cancers and, based on the gender, there was stark difference in occurrence for BT cancers above 45 years in comparison to OT cancers. Our analysis has shown that there exist significant disparities in the occurrence of OT and BT cancers for both the gender in different age-groups. This finding suggests that different etiological risk factors might be involved in the causation of OT and BT cancers in our population. However, the trend in the number of cases of OT cancers was similar after 45 years of age.

It was noteworthy that OT cancer was seen in significant proportion of educated and highly qualified patients $(71.1 \%)$ of our population. Patient-education strategies are needed for effective screening programs for cancer (Mahon, 2000). This is significant, as any screening program for early detection of OT cancer will not be hindered by poor literacy levels of patients and can be effectively intervened for early detection by screening program and cancer awareness programs as well. Though, there still exist a large proportion of patients with OT cancer who were illiterate $(28.8 \%)$ in the present study.

It has been shown that $85 \%$ of OT cancers are diagnosed in stage III and IV disease (Krishnamurthy and Ramshankar , 2013). Our results have shown that approximately $77 \%$ of OT cancers were diagnosed in advanced stages. Relatively more cases of OT cancers in comparison to BT cancers were diagnosed in stage I and II (early staged), ie $23.3 \%$ in OT cancer versus $9.2 \%$ in BT cancer. Currently there are no sustained and organized screening programs for oral cancers in our population, so such program can effectively improve the early detection of oral tongue cancers which will lead to a more number of OT cancers being diagnosed in earlier stages. Both base of tongue and oral tongue are richly supplied by lymphatics. So, early lymphatic spread that leads to stage III due to N1 nodal spread and stage IV in $\mathrm{N} 2 / \mathrm{N} 3$ nodal spread is commonly encountered in these cancers. Furthermore, the pattern of lymphatic spread to the neck nodes somewhat differs between oral tongue and the base of tongue. The presence of metastasis (M1) in BT and OT cancers was not so common at the time of diagnosis in the present study with a preponderance of distant metastasis in OT cancers $(2.5 \%)$ in comparison to BT cancers $(0.8 \%)$. For cancers of the OT there was a relative risk of 3 with BT cancer for distant metastasis, which was significant in the present analysis $(\mathrm{p}<0.05)$. The proportion of stage IV cases $(\mathrm{T} 4, \mathrm{~N} 2 / \mathrm{N} 3$ with $\pm \mathrm{M} 1)$ was similar for both OT and BT cancers. Tongue cancer is characterized by an unpredictable course despite adequate treatment. In spite of early stages, in cancers of the tongue, the prognosis is relatively poor (Vatanasapt et al., 2011). Identification of prognostic markers would enable clinicians to target patients who may benefit from a specifically tailored treatment strategy (Ferrari et al., 2009). Our analysis has shown that there is a probability of different clinical behavior for metastatic risk in cancer of OT and BT. However, further molecular and genetic characterization will be needed to for such differential behavior to be established in the cancers of the BT from OT cancer. A poor survival in tongue cancer with median survival of 20 months with one year survival of $61 \%$ has been shown (Sutandyo et al., 2014). There is disparity in survival due to HPV infection in oropharyngeal cancers with better 3-year survival rates for HPV positive cases (Ang et al., 2010). As most of the cases of tongue cancers in our study were anatomically located at the oropharynx so, it will be of interest to see the association of HPV with BT cancers in our population which will indicate better 


\section{Manigreeva Krishnatreya et al}

survival estimates for tongue cancers in our population as a whole.

In conclusion, our analysis has shown that in the sub sites of tongue cancers in our population, majority arose from the base of tongue. There were differences in the numbers of cases based on the age-group for gender in cancers of BT and the presence of distant metastasis at the time of diagnosis differs between cancer of OT and BT..

\section{Acknowledgements}

The National Centre for Disease Informatics and Research under Indian Council of Medical Research.

\section{References}

Ang KN, Harris J, Wheeler R, et al (2010). Human papillomavirus and survival of patients with oropharyngeal cancer. $N$ Engl $J$ Med, 363, 24-35.

Balaram P, Nalinakumari, KR, Abraham E, et al (1995). Human papillomavirus in 91 oral cancers from Indian betel quid chewers-high prevalence and multiplicity of infections. Int $J$ Cancer, 61, 450-4.

Chaturvedi AK (2014). Disparities in HPV-associated cancers. Paper presented at American Association for Cancer Research conference on the science of cancer health disparities, SA, Texas, USA

Coelho KR (2012). Challenges of the oral cancer burden in India. $J$ Cancer Epidemiol, 2012, 701932.

D’Souza G, Gross ND, Pai SI, et al (2014). Oral human papillomavirus (HPV) infection in HPV-positive patients with oropharyngeal cancer and their partners. J Clin Oncol, 32, doi 10.1200/JCO.2014.55.1341

Edge S, Byrd DR, Compton CC, et al (2010). AJCC Cancer Staging Manual. 7 th ed. Bangalore: Springer-Verlag.

Elango KJ, Suresh A, Erode EM, et al (2011). Role of human papilloma virus in oral tongue squamous cell carcinoma. Asian Pac J Cancer Prev, 12, 889-96.

Ferlay J, Soerjomataram I, Ervik M, et al (2014). GLOBOCON 2012: estimated cancer incidence, mortality and prevalence worldwide in 2012. IARC;WHO, Lyon. Available at http:// globocan.iarc.fr

Ferrari D, Codeca C, Fiore J, et al (2009). Biomolecular markers in cancer of the tongue. J Oncol, 2009, 412908

Herrero R, Castellsagué X, Pawlita M, et al (2003). Human papillomavirus and oral cancer: the international agency for research on cancer multicenter study. J Natl Cancer Inst, 95, 1772-83.

Kreimer AR, Clifford GM, Boyle P, Franceschi S (2005). Human papillomavirus types in head and neck squamous cell carcinomas worldwide: a systematic review. Cancer Epidemiol Biomarkers Prev, 14, 467-75.

Krishnamurthy A, Ramshankar V (2013). Early stage oral tongue cancer among non-tobacco users - an increasing trend observed in a south Indian patient population presenting at a single centre. Asian Pac J Cancer Prev, 14, 5061-65

Krishnatreya M, Hoque N, Sharma JD, Kataki AC (2014). Comparative study on the pattern of tobacco related cancers and habits of tobacco usage amongst bengali muslim community of Barpeta District Assam, India. Int Res $J$ Social Sci, 3, 22-4.

Mahon SM (2000). The role of the nurse in developing cancer screening programs. Oncol Nurs Forum, 27, 19-27.

National Cancer Registry Programme (2013).Three-year report of population based cancer registries:2009-2011. NCDIRICMR,Bangalore.
Ramshankar V, Soundara VT, Shyamsundar V, Ramani P, Krishnamurthy A (2014). Risk stratification of early stage oral tongue cancers based on HPV status and p16 immunoexpression. Asian Pac J Cancer Prev, 15, 8351-59.

Sutandyo N, Ramli R, Sari L, Soeis DS (2014). Profile and survival of tongue cancer patients in "Dharmais" cancer hospital, Jakarta. Asian Pac J Cancer Prev, 15, 1971-75.

Vatanasapt P, Suwanrungruang K, Kamsa-ard S, Promthet S, Parkin DM (2011). Epidemiology of oral and pharyngeal cancers in Khon Kaen, Thailand: a high incidence in females. Asian Pac J Cancer Prev, 12, 2505-08. 